\title{
Original
}

\section{Perioperative Complications with General Anesthesia Versus Local Anesthesia for Femoral Neck Fracture Operation}

\author{
Tomiaki IKeda, Kazuyuku Serada and Teruaki Tomaru
}

\begin{abstract}
This study compared the use of general versus local anesthesia in patients who underwent surgical operation for femoral neck fracture between 1992 and 1998 at Showa University Fujigaoka Rehabilitation Hospital. The patients were divided into a general anesthesia with oral intubation group and local anesthesia group (epidural anesthesia or spinal anesthesia). Complications during the perioperative period and the effects of anesthesia were compared between these groups. The results suggested that, in the selection of anesthetic methods, general anesthesia and local anesthesia have both advantages and disadvatages, and either of these methods can be selected. However, care must be taken to give optimal dosage of anesthetic agents and other drugs in anesthetic management because individual differences among elderly patients, who may have coexisting chronic disease, may influence the outcome.
\end{abstract}

Key words : anesthetic management, femoral neck fracture, general anesthesia, local anesthesia

\section{Introduction}

It was estimated in 1992 that there were 77,000 cases of femoral neck fracture per year in Japan ${ }^{1)}$. This figure is expected to gradually increase with the rapid increase in the elderly population in Japan. In the present study, we examined the complications during the perioperative period with different anesthetic methods on the outcome in the perioperative period in cases of surgical operation for femoral neck fracture.

\section{Materials and Methods}

A total of 44 elderly patients who underwent surgical operation for femoral neck fracture between 1992 and 1998 at Showa University Fujigaoka Rehabilitation Hospital were enrolled. The patients were divided into two groups; Group A, which included 22 cases of general anesthesia with oral intubation, and group B which included 22 cases who received local anesthesia (spinal or epidural anesthesia). We determined the preoperative, intraoperative, and postoperative complications, intraoperative bleeding volume, duration of anesthesia, duration of operation, surgical technique, number of days to start of oral food intake after operation, the time to administration of analgesic after operation, and requirement for blood transfusion in all patiens.

Department of Anesthesiology, Showa University Fujigaoka Hospital, 1-30 Fujigaoka, Aoba-ku, Yokohama-shi, Kanagawa 227-8511, Japan 
Epidural anesthesia was limited to anesthesia during operation using $1 \%$ or $2 \%$ mepivacaine. For spinal anesthesia, $0.3 \%$ dibucaine or $0.5 \%$ bupivacaine was used. In all cases, no drugs other than local anesthetic agent were given into the epidural space or subarachnoid space. In the general anesthesia with oral intubation group, anesthesia was induced with thiopental or propofoul. As a muscle relaxant, vecuronium or succinylcholine was used where appropriate, and anesthesia was maintained with oxygen $(2 \mathrm{l} / \mathrm{min})$, nitrous oxide $(41 / \mathrm{min})$ and sevoflurane $(1-2 \%)$.

Restlessness during night time, self-contradictory or inconsistent behavior, or selfextubation of the inserted needle from peripheral vein root were defined as dementia-like complications. If antihypertensive agents were administerd during operation, this was defined as hypertension. Where vasopressor was given, the complication was defined as hypotension.

For statistical analysis, a nonparametric one-way analysis test and chi-square test were used. A value of $\mathrm{p}<0.05$ was regarded as the level of significance.

\section{Results}

No significant difference was noted in age, sex, body weight, height, intraoperative bleeding volume, duration of operation, or duration of anesthesia between the two groups ( Table 1).

Preoperative complications were found in 37 cases, and there were generally 2 to 3 different types of coexistent disease in any one patient. These complications included 17 cases of hypertension, 14 cases of cerebrovascular disorder, and 8 cases of ischemic heart disease. There were many cases of hypertension and cerebrovascular disorder. The intraoperative complications included 7 cases of hypertension, 3 cases of hypotension in the systemic anesthesia group, and 2 cases of hypertension and 9 cases of hypotension in the local anesthesia group. The intraoperative incidence of hypertension was significantly higher in Group A than in Group B, but the intraoperative incidence of hypotension was significantly lower in Group A. In the general anesthesia group, cases of hypertension were frequently found during intubation and operation. There were also many cases of

Table 1 Patient Characteristics

\begin{tabular}{lcc}
\hline & A group & B group \\
\hline Sex (M/F) & $3 / 19$ & $4 / 18$ \\
Age & $76.6 \pm 2.4$ & $78.4 \pm 2.2$ \\
Height $(\mathrm{cm})$ & $152.5 \pm 1.7$ & $149.3 \pm 1.1$ \\
Weight $(\mathrm{kg})$ & $47.8 \pm 10.2$ & $44.1 \pm 7.7$ \\
Blood loss (ml) & $249.5 \pm 41.2$ & $229.6 \pm 52.6$ \\
Duration of operation (min) & $74.9 \pm 4.1$ & $75.6 \pm 6.5$ \\
Duration of anesthesia (min) & $132.8 \pm 5.6$ & $129 \pm 8.2$ \\
Site of operation & & \\
$\quad$ Median fracture of femoral neck & 14 & 11 \\
$\quad$ Fracture of femoral trochanter & 8 & 10 \\
Blood transfusion & 12 & 13 \\
\hline
\end{tabular}

Values are means \pm SEM.

A Group : General anesthesia group. B Group : Local anesthesia group 
Table 2 Complications

\begin{tabular}{llcc}
\hline & & A group & B group \\
\hline Preoperative complication & Hypertension & 8 & 9 \\
& Cerebrovascular disorder & 8 & 6 \\
& Ischemic heart disease & 5 & 3 \\
& Anemia & 6 & 7 \\
& Others & 9 & 8 \\
Intraoperative complication & Hypertension & $7^{*}$ & 2 \\
& Hypotension & $3^{*}$ & 9 \\
& Others & 2 & 3 \\
& Pnuemonia & 2 & 2 \\
& Dementia & 3 & 1 \\
& Others & 2 & 2 \\
\hline
\end{tabular}

Values are means \pm SEM.

A Group : General anesthesia group. B Group : Local anesthesia group

* Indicates $\mathrm{P}<0.05$

Table 3 Number of days to the start of oral food intake after operation (days) and time to the administration of analgesic after operation ( $\mathrm{min}$ )

\begin{tabular}{lcc}
\hline & A group & B group \\
\hline Number of days to the start of oral food intake after operation (days) & $1.35 \pm 0.21$ & $1.05 \pm 0.05$ \\
\hline Time to the administration of analgesic after operation ( $\mathrm{min})$ & $57.7 \pm 12.0$ & $98.3 \pm 31.5$ \\
\hline
\end{tabular}

Values are means \pm SEM.

A Group : General anesthesia group. B Group : Local anesthesia group

hypotension from the time after the induction of anesthesia to the start of operation.

In the local anesthesia group, there was a high incidence of hypotension during the operation. Many of these patients had anemia with a hemoglobin level of less than 9.0 $\mathrm{g} / \mathrm{dl}$ before the operation. In approximately $50 \%$ of cases, intraoperative or postoperative blood transfusion was performed. The postoperative complications included 4 cases of pneumonia and 4 cases of dementia (Table 2).

There were no differences between the two groups in number of days to the start of oral food intake after operation or administration of analgesic after operation (Table 3).

\section{Discussion}

Fracture of the femoral neck is generally divided in to medial fracture of the femoral neck, i.e. subcapital fracture in the joint capsula, and fracture of the femoral trochanter with the main fracture line on the femoral trochanter. Fracture of the femoral trochanter has been reported to occur 4 to 5 times more frequently than medial fracture ${ }^{2)}$, however in our institution medial fracture was more common than fracture of the femoral trochanter (Table 1). This may be attributed to the fact that we only examined a small number of patients, all of whom underwent operation.

The preoperative complications among the elderly patients are characterized by two or 
more different types of coexistant diseases that are usually chronic, and are often difficult to accurately identify ${ }^{3)}$. In this study preoperative complications were found in 37 cases, with 2 to 3 different types of coexistent disease. In particular, there were many cases of hypertension and cerebrovascular disorder. This suggests a need for preoperative examinations to check for valvular diseases or cardiac function by less invasive echocardiography in addition to chest radiography and EEG in these patients.

Hypertension was frequently found as an intraoperative complication during intubation and operation in the general anesthesia group. There were also many cases of hypotension from the time after the induction of anesthesia to the start of operation. These results show that the stimulation of oral intubation and operation induced hypertension by activation of the sympathetic nervous system, and that anesthetic agents frequently induced hypotension by suppression of the circulatory system in elderly patients. In contrast, hypotension was prevalent in the local anesthesia group. Patients with hypotension often had anemia with hemoglobin levels of less than $9.0 \mathrm{~g} / \mathrm{dl}$ before the operation. For about one-half of the cases, intraoperative or postoperative blood transfusion was performed. In elderly patients, oxygen supply to systemic organs is often limited and more attention should be given to anemia in elderly than adult patients. In contrast, cerebral thrombosis often occurs when hemoglobin concentration is high, and the volume of transfused blood must be carefully regulated. If necessary, preoperative blood transfusion should be performed to prevent hypotestion during the operation. These results indicate that care must be taken to account for individual differences in the response to drugs among the elderly patients, and that anesthetic care must also be given with optimal dosage of anesthetic agents and other drugs.

In selecting the anesthetic method, local anesthesia, in particular epidural anesthesia, is suitable for eldely patients with decreased respiratory function. A previous report found that venous thrombosis occurs less frequently with this type of anesthesia ${ }^{4)}$. Spinal anesthesia is also convenient because anesthesia can be achieved in a very short time. However, it has the disadvantage that blood pressure often decreases among the elderly patients, who have lower reserve potency in the circulatory systems. Also, the response to circulatory agents is decreased. In addition, the effects are often uncertain due to deformation of the spine, and since the patient is conscious for the operation may cause considerable stress in the maintenance of physical posture. Patients with dementia may also refuse to cooperate ${ }^{5)}$. However, some authors have described that it is advantageous because general anesthesia is less invasive in the lower limbs even among the eldery patients, and the patients can sit upright from day following operation ${ }^{6,7)}$. Thus, atelectasis, pneumonia or pulmonary infection can be avoided ${ }^{8)}$. Also, in our institution, there was no difference between the two groups in postoperative complications and intraoperative bleeding volume. Nor was a difference noted in the number of days up to the start of oral food intake and administration of analgesic. The results of our study suggest that, in the selection of anesthetic methods, both general anesthesia and local anesthesia have advantages and disadvantages, and that either of these methods can be selected with careful anesthetic management.

Finally, for femoral neck fracture among the elderly patients the possible risks must be realised. If indicated, full explanation should be given to the patient and the family on complications and prognosis and complete agreement should be obtained from them. 


\section{References}

1) Hayafune $T$ : Femoral neck fracture in elderly patients. Clin Orthop, $45: 448-451$ (1994)

2) Smith TC: Anesthesia and orthopedic surgery. In: Clinical Anesthesia, Barash PG (Ed), 2nd ed. J. B. Lippincott, Philadelphia, pp 1215-1235 (1992)

3) Stehling LC: Anesthetic consideration for the patient with fractured hip. In: Anesthesia for Orthopedic Surgery. Zauder HL (Ed), F. A. Davis, Philadelphia, pp 29-66(1980)

4) Sorenson RM and Pace NL: Anesthetic techniques during surgical repair of femoral neck fractures. Anesthesiology, $7:$ 1095-1104 (1992)

5) Berggren D : Postoperative confusion after anesthesia in elderly patients with femoral neck fracture. Anesth Analg, 66 : 479-504 (1988)

6) White IWC and Chappell WA : Anesthesia for surgical correction of fractured femoral neck. Anaesthesia, 32: 1107-1110 (1980)

7) McLaren AD and Reid VT: Anaesthetic techniques for surgical correction of fractured neck femur. Anaesthesia, 33 : 10-14 (1981)

8) Covert CR and Fox GS. Anesthesia for hip surgery in the elderly. Can J Anesth, 39 : 311-319 (1989)

[Received February 1, 1999 : Accepted March 26, 1999] 